\title{
In vitro and in vivo silencing of plasmodial dhs and elf-5a genes in a putative, non-canonical RNAi-related pathway
}

Andreas Schwentke ${ }^{1}$, Marcel Krepstakies ${ }^{2}$, Ann-Kristin Mueller ${ }^{3}$, Christiane Hammerschmidt-Kamper ${ }^{3}$, Basma A Motaal'2, Tina Bernhard', Joachim Hauber ${ }^{2}$ and Annette Kaiser ${ }^{1 *}$

\begin{abstract}
Background: Deoxyhypusine synthase (DHS) catalyzes the first step in hypusine biosynthesis of eukaryotic initiation factor 5A (elF-5A) in Plasmodium falciparum. Target evaluation of parasitic DHS has recently been performed with CNI-1493, a novel selective pro-inflammatory cytokine inhibitor used in clinical phase II for the treatment of Crohn's disease. CNI-1493 prevented infected mice from experimental cerebral malaria by decreasing the levels in hypusinated elF-5A and serum TNF, implicating a link between cytokine signaling and the hypusine pathway. Therefore we addressed the question whether either DHS itself or elF-5A is required for the outcome of severe malaria. In a first set of experiments we performed an in vitro knockdown of the plasmodial elF-5A and DHS proteins by RNA interference (RNAi) in 293 T cells. Secondly, transfection of siRNA constructs into murine Plasmodium schizonts was performed which, in turn, were used for infection.

Results: 293 T cells treated with plasmodial DHS- and elF-5A specific siRNAs or control siRNAs were analyzed by RT-PCR to determine endogenous $d$ hs -and elF-5A mRNA levels. The expressed DHS-shRNA and EIF-5A-shRNA clearly downregulated the corresponding transcript in these cells. Interestingly, mice infected with transgenic schizonts expressing either the elF-5A or dhs shRNA showed an elevated parasitemia within the first two days post infection which then decreased intermittently. These results were obtained without drug selection. Blood samples, which were taken from the infected mice at day 5 post infection with either the expressed EIF-5A-shRNA or the DHS-shRNA were analyzed by RT-PCR and Western blot techniques, demonstrating the absence of either the hypusinated form of elF-5A or DHS.
\end{abstract}

Conclusions: Infection of NMRI mice with schizonts from the lethal P. berghei ANKA wildtype strain transgenic for plasmodial elF-5A-specific shRNA or DHS-specific shRNA resulted in low parasitemia 2-9 days post infection before animals succumbed to hyperparasitemia similar to infections with the related but non-lethal phenotype $P$. berghei strain NK65. RT-PCR and Western blot experiments performed with blood from the transfected erythrocytic stages showed that both genes are important for the proliferation of the parasite. Moreover, these experiments clearly demonstrate that the hypusine pathway in Plasmodium is linked to human iNos induction.

\footnotetext{
* Correspondence: annette.kaiser@uk-essen.de

${ }^{1}$ University Duisburg-Essen, Medical Research Centre, Institute of

Pharmacogenetics, Hufelandstrasse 55, 45147 Essen, Germany

Full list of author information is available at the end of the article
} 


\section{Background}

RNA interference (RNAi) is an evolutionary conserved mechanism found across a range of eukaryotes, where it plays a key role in post-transcriptional gene regulation and protection of genomes. The process of RNAi is triggered by the recognition of double-stranded RNA (dsRNA), which is then processed into 21-25 nucleotide sequences by Dicer, a cytoplasmic dsRNA specific RNaseII endonuclease [1]. The generated RNAs associate with an RNA-induced silencing complex (RISC) and unwind in a strand-specific manner [2]. The resulting short interfering RNAs (siRNAs) then target homologous mRNA for degradation in combination with the RNase $\mathrm{H}$ enzyme Argonaute (Slicer) [3]. The stage of double stranded (ds) RNA processing may be surpassed by experimentally introducing sequence-specific siRNAs directly into cells.

Given the immense Public Health costs for malaria disease and the need for new drug targets a silencing approach employing RNAi might be extremely beneficial for the development of novel and advanced therapeutic strategies. Moreover, the ability to use RNAi for gene silencing in Plasmodium would provide a powerful means to gain insight into pathogenic blood stages.

Recent experiments performed by molecular genetics suggested that RNAi is not functional in malaria parasites [4]. These authors showed that expression of the analyzed proteins continued despite the application of a variety of RNAi-based strategies to target genes which are non-essential to either growth or development of P. falciparum or P. berghei. In good agreement, control experiments with Trypanosoma brucei, a protozoan parasite with validated RNAi, were successful. Furthermore, to determine whether a primitive RNAi machinery exists in Apicomplexa a comparative analysis of Apicomplexan and other protozoan genomes was undertaken. Taken together these data argued that RNAi is absent in malaria parasites [4].

Several studies, however, reported the successful application of RNAi for gene silencing in the erythrocytic stages of Plasmodium. A series of experiments has been performed by introducing long dsRNAs by electroporation into infected erythrocytes. Gissot and coworkers [5] performed silencing experiments with MybB1, a transcription factor in Plasmodium thereby demonstrating its essential role in the erythrocytic stage. Kumar and colleagues [6] showed in a similar manner the requirement of a serine-threonine phosphatase for DNA-replication in Plasmodium. Tuteja and colleagues [7] identified a signal peptidase that is required for intra-erythrocytic growth by RNAi. Apart from electroporation [8], siRNAs have also been added directly to the culture medium. Cysteine proteases falcipain-1 and falcipain-2, which are necessary for haemoglobin degradation, have been shown to be essential for the blood stages [9]. However, this finding is in question since standard disruption techniques showed no effect on parasitic development in the blood stages [10]. While the latter authors suggested RNAi to be functional in Plasmodium, most of these cases resulted in parasitic death or significant growth defects due to unspecific downregulation of multiple genes by RNAi.

Deoxyhypusine synthase (DHS) catalyzes the first step in the biosynthesis of the amino acid hypusine (Hyp), a novel amino acid present in eukaryotic initiation factor 5A (eIF-5A) to form the deoxyhypusinylated intermediate. DHS transfers the aminobutyl moiety from the triamine spermidine to the $\epsilon$-amino group of Lys ${ }^{50}$ present in the hypusine loop. Both genes have been identified in $P$. falciparum and P. vivax $[11,12]$. Hitherto, the biological function of this posttranslational modification is unknown. Recent studies have implicated a permissive role of eIF- $5 \mathrm{~A}^{\text {Hyp }}$ in various diseases. In diabetes type 2 pancreatic stressed $ß$-cells [13] and in HIV-infected $\mathrm{T}$ cells, eIF- $5 \mathrm{~A}^{\text {Hyp }}$ is functional as a nucleocytoplasmic shuttle protein for the transport and translation of specific mRNAs [14].

Particularly in HIV, eIF-5 $\mathrm{A}^{\text {Hyp }}$ is essential for the nucleocytoplasmic transport and translation of incompletelyspliced mRNAs encoding viral proteins $[15,16]$. In diabetes type 2 eIF- $5 \mathrm{~A}^{\text {Hyp }}$ enables cytokine-mediated islet dysfunction through the direct posttranscriptional regulation of the mRNA encoding iNos2 (Nos2) in both rodent and human cells $[13,17]$. Importantly, the immunological events which lead to severe malaria are complex and parallel events present in HIV-infection and pancreatic stressed $\beta$-cells. Exogenous NO administration $[18,19]$ prevents the syndrome of severe malaria. Since a parasite specific nitric oxide synthase does not exist, the defense response may be attributed to the host specific iNos.

Cerebral malaria $(\mathrm{CM})$ is characterized by clinical features like cognitive dysfunctions, seizures, coma and clinical parameters like anemia, metabolic acidosis, renal insufficiency and hypoglycaemia. Although the understanding of malaria pathogenesis is rudimentary, different theories have been accepted to understand the pathological process [20]. The sequestration theory suggests that seizures might be caused by the adherence of parasites to red blood cells and subsequent expression of parasite specific antigens which in turn lead to obstruction of blood flow, cerebral hypoxia and decreased removal of waste. For the neurological symptoms there is growing evidence that parasite-induced sequestration of infected and uninfected erythrocytes changes bloodbrain barrier function. Moreover, host-specific immune mechanisms may be important in response to the presence of parasites in the CNS.

In a first step of the infection process parasitized red blood cells adhere to brain microvascular endothelial 
cells through the erythrocyte membrane protein [21]. After release of merozoites parasitic glycosylphosphatidylinositol (GPI) is released which induces a local inflammatory response involving natural killer and subsequently $\mathrm{CD} 4^{+} \mathrm{T}$ cells. At this stage of the infection, proinflammatory cytokines including tumor necrosis factor $\alpha$ (TNF- $\alpha$ interferon $\gamma$ (IFN- $\gamma$ and interleukin (IL)-1ß are produced locally before the entry of the systemic phase in which cytokines activate macrophages and $\mathrm{CD}^{+} \mathrm{T}$ cells [21]. In the systemic phase, more platelets and microparticles are released inducing perforinmediated lesions in the endothelium [21].

Recently, metabolic changes in the central nervous system caused by the parasite, have been characterized as a third theory in explaining the pathology of malaria. During CM an increase of lactate and alanine concentration and alterations in tryptophane metabolites like the kynurenine pathway lead to an increased permeability of the blood brain barrier for plasma proteins.

DHS has been recently validated as a druggable target by the small molecule CNI-1493, a synthetic guanylhydrazone [22], which significantly extends the survival rate of Plasmodium berghei ANKA-infected C57BL/6 mice [22]. Initial studies with the compound suggested that the mechanism of action can be attributed to the inhibition of parasitic DHS and the translation of host specific TNF $\alpha$-mRNA [23], indicating a link between host cell proinflammatory cytokine production and the hypusine pathway.

To study the outcome after an in vivo knockdown of this enzyme and its target protein eIF-5A in the erythrocytic stages of Plasmodium in more detail, we transfected siRNA constructs targeted to both genes based on in vitro knockdown experiments into $P$. berghei ANKA schizonts, using standard transfection methods [24].

\section{Results}

In vitro knock-down of P. falciparum DHS and elF-5A by RNAi

Two different DHS short hairpin RNAs (shRNAs), \#43 and \#176 (see Materials and Methods section), expressed from the pSilencer1.0-U6 vector were applied to knock down the DHS protein from P. falciparum. The shRNA \#43 targets the dhs sequence at nucleotide positions 337-358, while shRNA \#176 targets the dhs sequence at nucleotide positions 1269-1290 within the P. falciparum mRNA. Both constructs were individually cotransfected with plasmodial DHS expression vector into $293 \mathrm{~T}$ cells to verify the expected degradation of the $d h s$ transcript. The results obtained by RT-PCR analysis show a significant knock-down of plasmodial dhs transcript by the shRNA P \#176 construct (Figure 1A, lane 4), as opposed to when the shRNA P \#43 was expressed (lane 5). By contrast, a control siRNA which lacks complementary sequences in the human genome did not negatively affect the abundance of the Plasmodium transcript with the expected size of 612 bp (amino acid positions 208412) (lane 1). To exclude any off-target effects a number of control experiments was performed. As a positive control the recombinant plasmodial DHS expression vector was transfected alone into 293T cells. Following RT-PCR the cDNA fragment of 612 bp was detected (lane 3). No transcript could be observed when untransfected 293Tcells were analyzed (lane 2). Next, we amplified the human GAPDH sequence, representing a housekeeping gene, to control the various cotransfections. As shown, the presence of the expected GAPDH amplificate was detected in all analyzed samples (Figure 1B), suggesting that the silencing effect of the DHS siRNA used is specific since the dhs amplificate does not show any homology to its human orthologue. In a separate set of experiments we applied 4 different shRNAs to knock down the eIF-5A precursor protein. The $p$ Silencer1.0-U6 vectors expressing different eIF-5A shRNAs (\#5, \#6, \#7, and \#18; see Materials and Methods and (Additional file 1: Figure S1) were individually cotransfected with plasmodial eIF-5A expression vector into $293 \mathrm{~T}$ cells. Again, the monitoring of eIF-5A transcript abundance was performed by RT-PCR. From the 4 tested eIF-5A siRNAs only shRNA \#18 (Figure 2A, lane 3) was capable of completely downregulating the plasmodial eIF-5A mRNA level in 293T cells. For all other constructs an in vitro knockdown was unsuccessful (our own data; not shown).

Control reactions with non-transfected cells (Figure 2A, lane 1) and eIF-5A shRNA \#18 cotransfected with the aquaporin-specific siRNA (Figure 2A, lane 4) did not change the silencing effect. Although eIF-5A is a highly conserved protein in eukaryotes its nucleic acid sequence is significantly divergent in comparison to its human orthologue and thus amplificates from endogenous eIF-5A are not expected. Again, we monitored the presence of GAPDH by RT-PCR in all transfections (Figure 2B) independently of the presence of the siRNA construct.

To further validate the RT-PCR experiments the limit of detection for the corresponding mRNAs i.e. eIF-5A and $d h s$ was determined. Titrated dhs-specific mRNA resulted in a limit of detection of $20 \mathrm{ng}$ while eIF-5Aspecific mRNA could only be detected at a concentration of $200 \mathrm{ng}$. Optimal primer binding was determined for eIF-5A-specific primers at a cDNA concentration of $130 \mathrm{ng}$ and for $d h s$-specific primers at a cDNA concentration of $650 \mathrm{ng}$ (data not shown).

In sum, these data demonstrated that Plasmodiumspecific eIF-5A and DHS sequences can in principal be silenced by RNAi. 

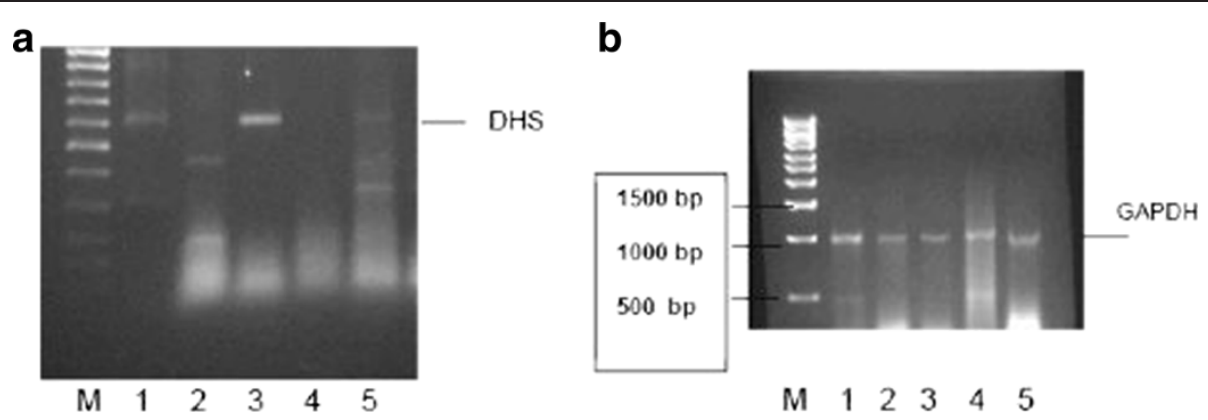

Figure 1 A) Inhibition of plasmodial DHS by RNAi and monitoring of the 612 bp amplificate by RT-PCR after transfection of 293 T cells with the DHS expression vector. 293T cells were cotransfected with: 1) Scramble II-duplex shRNA; 2) no transfected DNA; 3) the recombinant pcDNA3 vector containing 612 bp of a -highly conserved region of the dhs gene from $P$. falciparum (amino acid positions 208-412); 4) DHS- shRNA construct P\#176; 5) DHS- shRNA construct P\#43. B) Analysis of the 983 bp GAPDH amplificate in the cotransfected 293T cells described in Figure $1 \mathrm{~A}$.

\section{Monitoring in vivo silencing of elF-5A and DHS in erythrocytic stages after infection of NMRI mice with transgenic schizonts from $\mathrm{P}$. berghei}

With regard to the in vitro results, we investigated the silencing effect of the expressed DHS-specific and eIF-5A specific shRNAs in an in vivo rodent model of $P$. berghei ANKA strain [24]. Infection of NMRI mice with $\mathrm{P}$. berghei ANKA wild type strain leads to experimental cerebral malaria within 6 to 10 days p. i. although the parasitemia is only in the range of $3-5 \%$ infected erythrocytes. In case of the infectious but non lethal phenotype $P$. berghei strain NK56, the infected mice succumb to high parasitemia within 80 days p.i. without cerebral malaria.

In a first step DHS-specific shRNA \#176 or eIF-5Aspecific shRNA \#18 expressed from pSilencer 1.0-U6 vector was transfected into schizonts, the late developmental stage of the parasite. These transgenic schizonts were applied to NMRI mice for infection. In vivo gene silencing was monitored in the animals' erythrocytes at day 2 post infection by RT-PCR as before. Infection with schizonts containing the eIF-5A-specific shRNA \#18 vector (Figure 3A lane 2) led to a complete disappearance of the respective transcripts, at least within the detection level of this assay. By contrast, the eIF-5A sequences were clearly detected in the erythrocytic stage after infection with schizonts, which were transfected with the $d h s$-specific shRNA \#176 vector (Figure 3A, lane 1). Several control reactions were applied. The RT-PCR reactions of a kanamycin control RNA of $1.2 \mathrm{~kb}$ (Figure 3A, lane 5) and that of the recombinant eIF-5A plasmid from $P$. vivax was monitored, resulting in amplification products of approximately $323 \mathrm{bp}$ and $448 \mathrm{bp}$, respectively (Figure 3A, lanes 5 and 4). In parallel we confirmed the quality of the total cellular RNA preparation for the presence of the $\alpha$-tubulin II sequences, which are expressed in the asexual blood stages of Plasmodium (lane 4).

Next we monitored the effect of in vivo eIF-5A silencing on the protein level. As shown in Figure 3B, eIF-5A protein was absent in NMRI infected mice with transgenic schizonts expressing the \#18 eIF-5A-specific shRNA. In these experiments a polyclonal anti-eIF-5A antibody raised against the highly conserved $P$. vivax protein (96\% identity) was used. NMRI mice infected with transgenic schizonts expressing the \#176 DHS-

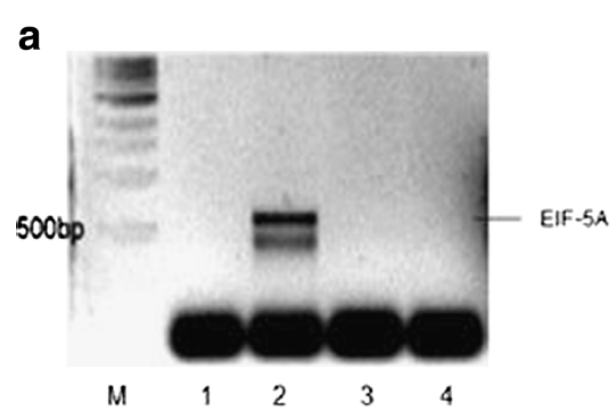

b

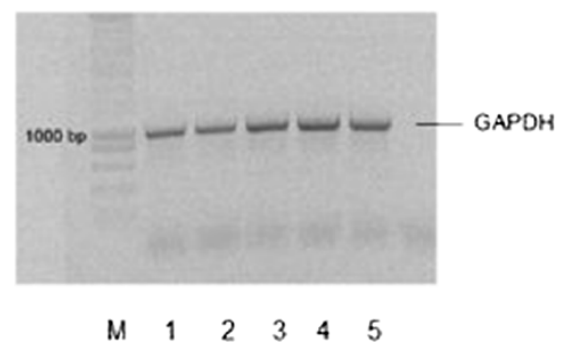

Figure $\mathbf{2}$ A) Silencing of parasitic ElF-5A by RNAi in 293 T cells and subsequent monitoring by RT-PCR. A cotransfection was performed with: 1) no transfected DNA; 2) recombinant, plasmodial elF-5A expression plasmid with the 483 bp cDNA; 3) EIF-5A-shRNA construct P\#18; 4) aquaporin-5-specific siRNA. B) The 983 bp GAPDH amplificate was used as an internal control in the transfected mammalian cell line. 


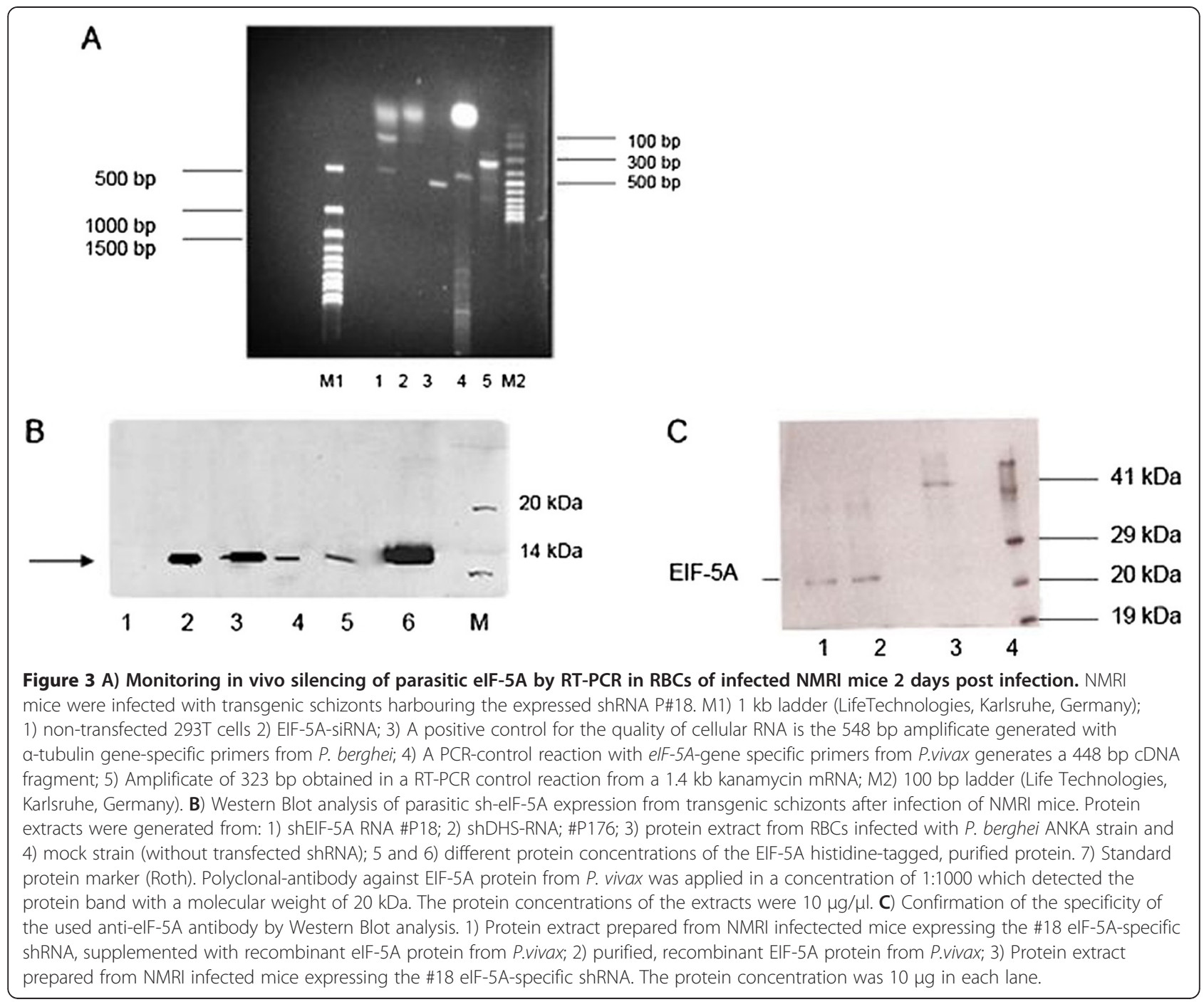

specific shRNA construct showed that the unmodified or hypusinated eIF-5A protein was present (Figure 3B, lane 2). This result implies that the \#176 DHS-specific shRNA construct exclusively affects the DHS protein. Although eIF-5A is not modified and is mostly abundant in its unhypusinated form, it is recognized by the polyclonal anti-eIF-5A antibody (Figure 3B, lane 2). The results further support the observation that the RNA encoding the eIF-5A gene is present in the erythrocytic stages after infection with schizonts expressing the DHS-shRNA \#176 (Figure 3A, lane 4). The polyclonal antibody detected the eIF-5A protein with a size of $17,75 \mathrm{kDa}$ in the P. berghei ANKA strain (Figure 3B, lane 3 ) as well as in the mock control strain (Figure 3B, lane 4), while the eIF-5A protein from $P$. vivax displayed the expected molecular mass of approximately $20 \mathrm{kDa}$ (lanes 5 and 6).

To further support the specificity of the polyclonal anti-EIF-5A antibody, protein extracts obtained from the infected NMRI mice expressing the \#18 eIF-5A-specific shRNA were spiked with purified eIF-5A protein from $P$. vivax (Figure $3 \mathrm{C}$, lane 1 ). The $P$. vivax anti-eIF5A antibody clearly detected the EIF-5A protein in the respective extract (lane 1) while EIF-5A protein was absent in the crude extract of $P$. berghei ANKA strain (Figure 3C, lane 3). Moreover, the purified, recombinant eIF-5A protein was clearly recognized by the antibody (Figure 3C, lane 2). These data suggest that an in vivo knockdown of eIF-5A is possible.

A DHS-specific RT-PCR was performed to control formation of the 1248 bp cDNA fragment in the erythrocytic stages after infection of NMRI mice with transgenic schizonts harbouring the DHS-shRNA \#176 and the eIF-5A \#18 construct (Figure 4A, lanes 1-2). A dhs-specific transcript was not detectable in the \#176infected (shRNA expressing) erythrocytes (lane 1), while it was present in the \#18-infected (shRNA expressing) erythrocytes (lane 2) and in the control reaction with 


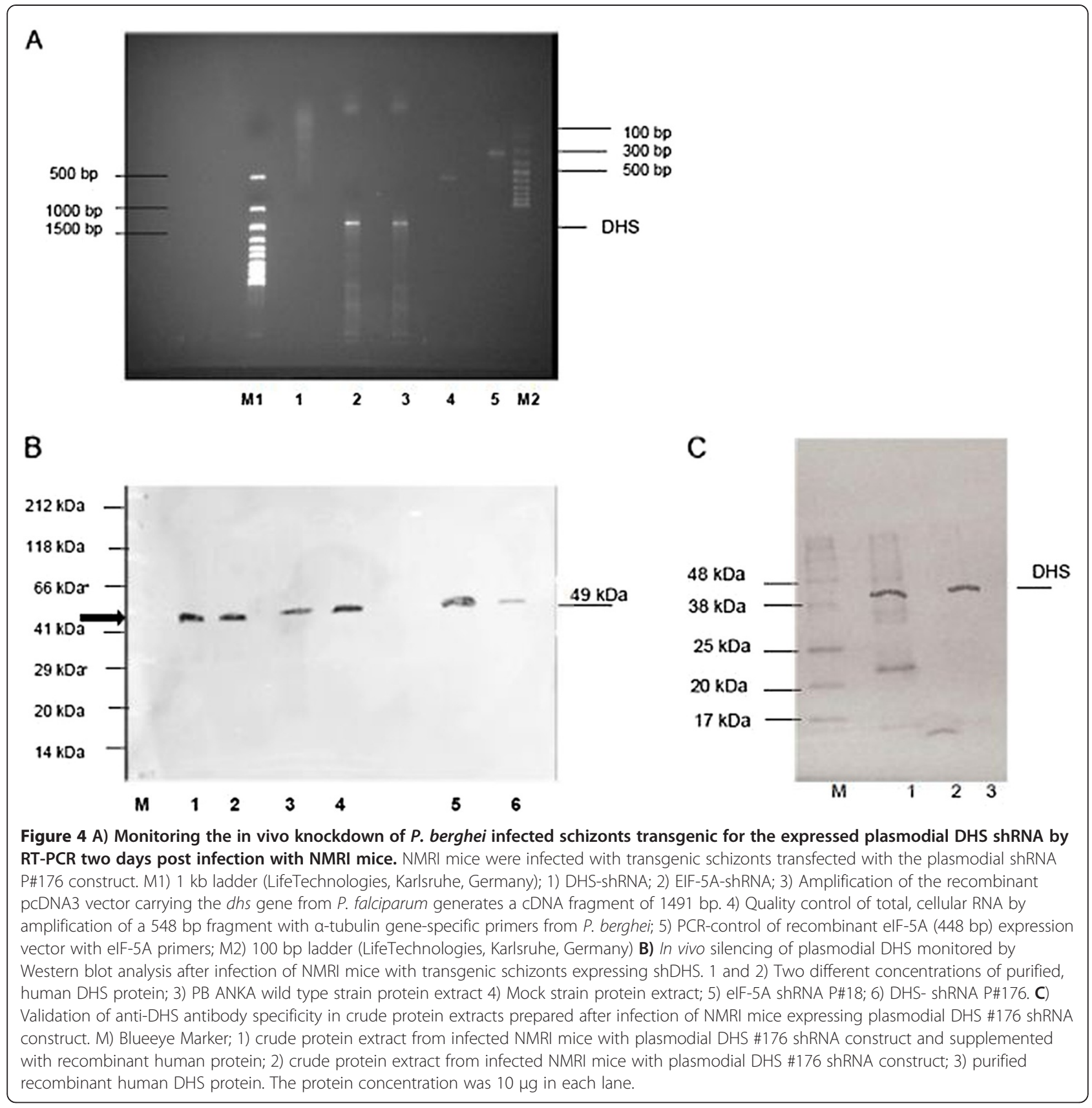

plasmodial $d h s$-specific primers (lane 3). Additionally, the quality of the cellular RNA was confirmed with $P$. berghei specific $\alpha$-tubulin primers (lane 4) by reverse transcription using a $1.2 \mathrm{~kb}$ Kanamycin-mRNA (lane 5). In parallel we controlled in vivo silencing of DHS levels by Western blot analysis (Figure 4B). A polyclonal antiDHS antibody raised against the human DHS protein detected the predicted size of $41 \mathrm{kDa}$ when different concentrations of purified human DHS were applied (lanes 1 and 2). Results from an amino acid alignment showed that human DHS isoform1 shares $57 \%$ amino acid identity to the P. falciparum 3D 7 orthologue, 58\% amino acid identity to the P. vivax orthologue and $56 \%$ identity to $P$. berghei. These highly conserved amino acid regions were apparently recognized by the human antibody. Protein extracts prepared after infection with P. berghei (lane 3) and mock strain (lane 4) showed the expected $49 \mathrm{kDa}$ orthologue of DHS. DHS was completely abundant in the eIF-5A-shRNA mutant \#18 (lane 5) and a faint band was visible in the DHS-shRNA mutant (lane 6), although no cDNA could be detected in a RT-PCR reaction.

Again, as already performed with eIF-5A, the specificity of the human anti-DHS antibody was confirmed. 
Protein extracts prepared from the infected NMRI mice harbouring the expressed sh-RNA construct \#176 were supplemented with recombinant, human DHS protein (Figure 4C, lane 1). The human anti-DHS antibody clearly detected the recombinant human protein (lane 3 ) and the added DHS protein (lane 1). However, in the extract with the plasmodial shRNA \#176 a DHS signal was absent (lane 2). These data demonstrate the validity of this antibody.

\section{Monitoring parasitemia after infection of schizonts transfected with elF-5A- and DHS-specific siRNA}

With respect to the in vitro silencing data, $P$. berghei purified schizonts were transfected with either the eIF-5A shRNA construct (P \#18) or the DHS shRNA (P \#176) construct. In both cases, transfected cells were tracked for infection in recipient outbred NMRI mice without any selection pressure.

In two independent, different sets of experiments infection of mice was monitored after transfection of recombinant schizonts expressing either the P \#176 DHSshRNA, or the $\mathrm{P} \# 18$ construct (eIF-5A-shRNA) (Figure 5). As a control, an infection was performed using a mock strain, which was not transfected with DNA. From day 2 to day 10 post infection, parasitemia was significantly lower in both lines compared to the untransformed mock strain. By contrast, the mock strain displayed a parasitemia of $9 \%$ at day 6 post infection, compared to the transfected parasites with the DHS-shRNA (4.5\%) or the eIF-5AshRNA. After 9 days post infection, parasitemia increased significantly in both infection experiments, harbouring either the transgenic schizonts with the DHS-shRNA or the eIF-5A-shRNA.

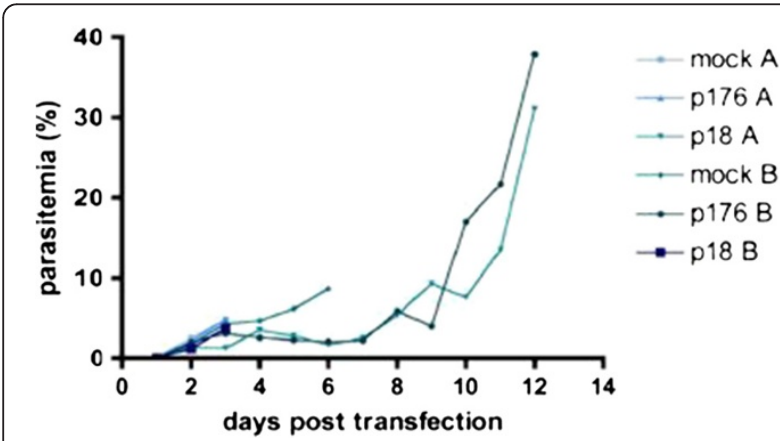

Figure 5 Parasitemia of outbred infected recipient mice post transfection with schizonts transgenic for parasitic elF5AshRNA or DHS-shRNA. Infection with each construct was performed in two different independent experiments with two mice per condition. Pale blue triangles and blue points represent the curves for the determined parasitemias post infection with the shDHS P\#176 in two mice. Pale blue upside down triangles and blue squares represent the monitored parasitemia with the expressed elF5A-sh P\#18. The parasitemia for the mock control strain is represented by the pale blue dot and the pale blue rhomb.
Studies on the effect of cytokines on iNos production due to posttranslational modification of elF-5A

To investigate a possible link between cytokine signaling and translation of iNos 2 mRNA of the host, Western blots were performed with protein extracts from serum of the infected erythrocytic stages of NMRI mice. Figure 6 shows that signals for iNos2 were absent in serum p. i. after DHS silencing with construct P \#176 and eIF-5A-shRNA construct P \#18 (Figure 6, lanes 1 and 2), while iNos2 protein with a molecular size of approximately $131 \mathrm{kDa}$ was detectable in the $P$. berghei ANKA strain infected erythrocytes (Figure 6, lane 3). Most notably, prominent signals for iNos2 protein were detected in immortalized T cells (Jurkat cells) (Figure 6, lane 4 uninduced and lane 5 induced) and a monocytic cell line (Mono Mac) (Figure 6, lane 6). No signal was obtained in HeLa cells (lane 7).

There was no difference in signal intensity between induced and uninduced cells probably due to the induction by ionomycin/PMA (phorbol 12-myristate 13acetate), which might not be the correct inductor to stimulate cytokine cell signaling.

To further support these results nitric oxide was quantified in a colorimetric assay after an enzymatic conversion of nitrate to nitrite by the enzyme nitrate reductase followed by detection of nitrite as a colored azo dye product. The amount of the formed nitrite and nitrate from nitric oxide was approximately 20 -fold lower in the serum after infection of mice with the shRNA construct P \#18 (108,8 $\mu \mathrm{M} / \mathrm{L})$ (Table 1) and 18-fold lower with the shRNA construct P \#176 (120 $\mu \mathrm{M} / \mathrm{L})$ (Table 1$)$ in comparison to the wild type $(2260,5 \mu \mathrm{M} / \mathrm{L})$.

\section{Discussion}

Hitherto, the biological function of the unusual amino acid hypusine has not been studied in Plasmodium. Previous studies showed that hypusination of eIF-5A is important for cell proliferation of the parasite [11]. Deoxyhypusine synthase (DHS), which catalyzes the first step in the hypusine pathway has been recently targeted by a small molecule inhibitor, CNI-1493, which blocked parasitic growth in vitro and in vivo [22]. Another excellent way to study the biological function of this posttranslational modification in more detail is a genetic analysis by loss of function of the proteins involved in hypusine biosynthesis.

For the future it will be an important issue to pursue a targeted, stable gene disruption of the dhs and eIF5 Agenes in Plasmodium, since their exact function in the erythrocytic life cycle stages is still unknown. To date gene disruption by insertion strategy has been successfully shown in the rodent model of $P$. berghei and it is partly working in the intraerythrocytic schizogeny of P. falciparum $[24,25]$. 


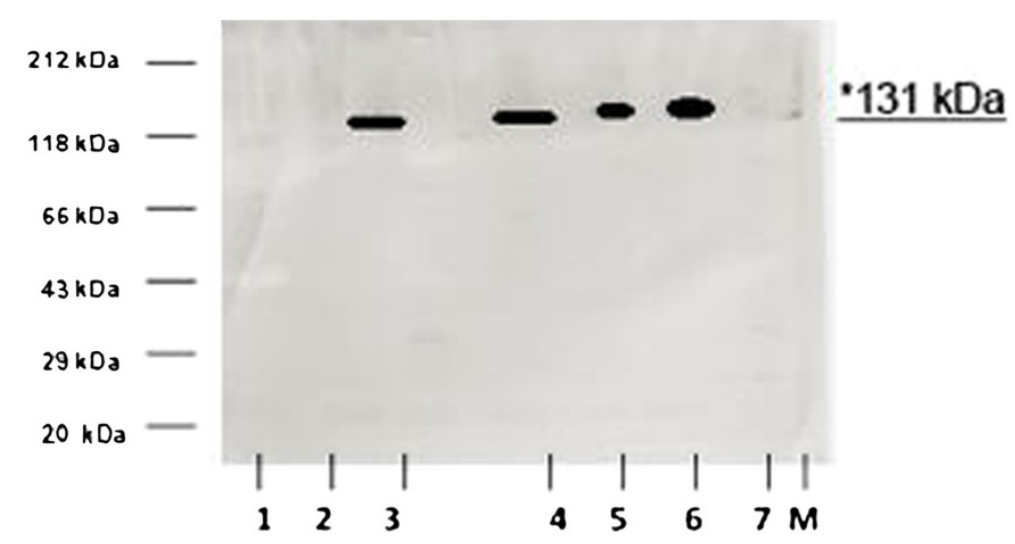

Figure 6 Cytokine signaling for human iNos2 translation is dependent on the hypusine pathway during the infection of Plasmodium. Western Blot analysis was performed with equal amounts of protein $(10 \mu \mathrm{g})$ extracted from the infected erythrocytic stages with transgenic schizonts from $P$. berghei ANKA strain 1) protein extract prepared from serum after infection with schizonts harbouring the expressed plasmodial DHS-shRNA or 2) the elF-5A-siRNA expression construct; 3) P. berghei ANKA strain; 4) induced and 5) non- induced Jurkat cells; 6) Mono Mac 1 cells; 7) HeLa cells; M) Standard protein marker Roth, St. Leon, Germany. Detection of the iNos2 protein with a molecular size of 131 kDa was performed with a human anti-Nos2 antibody in a dilution of 1:1000.

The understanding of cerebral malaria $(\mathrm{CM})$ pathogenesis is still rudimentary [26]. Our results clearly demonstrate that the hypusine pathway in Plasmodium supports at least two different hypotheses in the pathogenesis of cerebral malaria i.e. the sequestration theory and the inflammation hypothesis. One of the underlying mechanisms of cerebral malaria pathogenesis is the adherence of parasitized red blood cells to vascular endothelial cells by parasite specific proteins. Infected NMRI mice transfected with schizonts transgenic for plasmodial eIF-5A- or DHS-specific shRNA showed a $50 \%$ reduced parasitemia in comparison to the untransfected control within 2 to 9 days post infection. This may indicate the preventing of parasitic sequestration.

In a first approach to test the possibility whether a knockdown of DHS and its precursor protein eIF-5A is possible in Plasmodium, an in vitro knockdown by RNAi was performed since an unequivocal demonstration that the Plasmodium genome contains any of the conserved RNAi machinery genes or enzymes is to date missing. In the past, RNAi in circulating malaria parasites was

Table 1 Colorimetric determination of nitric oxide formation as nitrate and nitrite in sera from infected mice obtained after P.berghei ANKA strain infection and after infection with schizonts harbouring the expressed plasmodial DHS shRNA \#176 or plasmodial EIF-5A shRNA \#18

\begin{tabular}{ll}
\hline Nitrate and nitrite $[\boldsymbol{\mu m o l} / \mathbf{L}]$ & Wild type and transfectants \\
\hline 2200,5 & P. berghei ANKA wild type \\
\hline 120 & DHS-specific shRNA \# 176 \\
\hline 109 & ElF-5A-specific shRNA \# 18
\end{tabular}

Nitrate and nitrite determination after infection of mice with transgenic schizonts expressing plasmodial DHS and EIF-5A shRNAs. performed showing 50\% reduction at the expression level of berghepains which are homologues of cysteine proteases in Plasmodium [27]. For the siRNA experiments, a strategy to reduce gene expression in cultured cell lines with pSilencer1.0-U6 vectors producing the respective shRNAs from the U6 promotor was selected.

The data indicate that an in vitro knockdown of eIF$5 \mathrm{~A}$ with four different shRNAs was not completely ablating eIF-5A expression except for the shRNA \# P18 in $293 \mathrm{~T}$ cells (Figure 2A, lane 3) which markedly reduced the eIF-5A transcript level. These four shRNA constructs of eIF-5A were targeted all over the eIF-5A sequence. The eIF-5AshRNA \#18, which targets positions 163-184 in the eIF-5A nucleic acid sequence, caused a complete decrease in eIF-5A mRNA levels. These results are in agreement with the structural model of human eIF-5A1 [30], which consists of two domains, a basic N-terminal domain with the hypusine loop and an acidic -terminal domain connected by a hinge. Within the basic $\mathrm{N}$-terminus, the hypusine modification covers amino acid positions $46-54$ i. e. nucleic acid positions 138-162 which are very close to the 3 ' prime end of the hypusine loop.

By contrast eIF-5A shRNA \#7 targets position 115136, which is proximal to the 5'-end of the loop, does not affect mRNA abundance. It is likely that the secondary structure of the hypusine loop at this position might block the degradation of the specific mRNA [28]. Taken together, from four tested shRNAs, only one, the eIF5A-specific shRNA \#18 caused a considerable decrease of the eIF-5A transcript in vitro.

Two DHS-shRNAs, \#43 and \#176, targeting nucleotide positions from 337-358 bp and 1269-1290 bp, respectively, were employed for an in vitro knockdown of DHS 
from Plasmodium. Surprisingly, the DHS-shRNA construct \#176 was successful to downregulate the dhs transcript significantly (Figure 1A, lane 5), although the targeted sequence did not cover the active site of the enzyme within the amino acid region between Lys $^{287}$ and $\mathrm{Glu}^{323}[28,29]$.

Subsequently, monitoring of in vivo silenced $P$. berghei blood stage parasites transgenic for either eIF-5AshRNA or DHS-shRNA post transfection was performed by RT-PCR. In case of the eIF-5A-shRNA containing blood stages the eIF-5A transcript was not present (Figure 3A, lane 2), while in erythrocytes with the DHSshRNA (Figure 3A, lane 2) the dhs cDNA was not abundant (Figure 4A, lane 1). However, the eIF-5A transcript was detectable, suggesting that the silencing effect is rather specific.

Moreover, these results were confirmed by Western blot analysis where the $17,75 \mathrm{kDa}$ eIF-5A protein was absent in the transgenic $P$. berghei ANKA parasites harbouring the eIF-5A-specific siRNA. Both proteins, i.e. the $P$. falciparum and the $P$. berghei homolog share amino acid identities of $73 \%$. In a control experiment the antibody raised against the eIF-5A protein from $P$. vivax crossreacted with the eIF-5A homologue from the mock strain and the $P$. berghei ANKA strain resulting in a protein of $17,75 \mathrm{kDa}$ [30] (Figure 3B, lanes 3 and 4). To monitor suppressed DHS expression a polyclonal human antibody was applied which detected the $P$. berghei orthologue of $49 \mathrm{kDa}$ (Figure 4B, lanes 3 and 4) in the mock control and the P. berghei ANKA strain. By contrast a faint band was detected in the DHS siRNA mutant suggesting that the gene may not be silenced completely.

The inflammation hypothesis in cerebral malaria implies that brain damage is a result of the inflammatory response of the human host to the parasite in the central nervous system (CNS). The production of proinflammatory cytokines like IL-1 $\beta$, TNF- $\alpha$, IFN- $\gamma$ leads to secretion of nitric oxide which kills the parasite. It has been recently reported that hypusinated eIF-5A is required in part for the nuclear export and translation of iNosencoding mRNAs in pancreatic, stressed $ß$-cells after release of proinflammatory cytokines [17]. To test this hypothesis the host iNos2 protein levels were monitored in serum after infection with $P$. berghei ANKA strain and the two transfected shRNAs P \#176 (DHS) and P \#18 (EIF5A), since a parasitic nitric oxide synthase is absent. We could clearly demonstrate that in both mutants there is no response to cellular stress i.e. induction of inducible nitric oxide synthase (iNos2) of the human host once modification of eIF-5A is interrupted by silencing of either parasitic DHS or eIF-5A. However, nitric oxide synthase is induced 20 -fold after infection with the wild type $P$. berghei ANKA strain in comparison to the
shRNA mutants P \#176 (DHS) and P \#18 (EIF5A) with a 18-fold and 20-fold lowered formation of nitric oxide. These findings do not only prove a link between the hypusine pathway and iNos production but also broaden our understanding of the $\mathrm{CM}$ malaria pathology and implicate alternative strategies for therapy. Similar results have been obtained in DHS heterozygous knockout mice with attenuated cytokine signalling as evidenced by reduced nitric oxide synthase production [31].

Malaria patients often present with hypoargininemia [32], and metabolomic studies of Plasmodium falciparum during its $48 \mathrm{~h}$ intraerythrocytic life cycle reveal nearly complete depletion of L-arginine levels. Nitric oxide synthase is induced by arginine and catalyzes the reaction to nitric oxide (NO) and urea. However, in cerebral malaria there is a lack of nitric oxide due to the presence of parasite-specific arginase which leads to a depletion of arginine and subsequent downregulation of host-specific nitric oxide synthase. This may allow the parasite to evade a NO-dependent immune response in the host since NO is deleterious to parasite proliferation [33]. During Plasmodium berghei ANKA infection in mice exogenous nitric oxide decreases brain vascular inflammation, leakage and venular resistance $[17,18]$ and protects against cerebral malaria.

Finally, the crystal structure of Plasmodium arginase has been resolved recently and indicates a low complexity region [33] which is largely disordered and its deletion does not significantly compromise enzyme activity. Moreover, disruption of $P$. falciparum arginase led to an apparent reduction in liver stage infection.

\section{Conclusions}

Although it has been previously suggested that RNAi is not functional in Plasmodium, a putative, non-canonical RNAi pathway might exist in malaria parasites. In vivo knockdown of eIF-5A and DHS by expression of shRNAs after infection in a rodent model decreased parasitemia intermittently in the development of cerebral malaria. The data are similar to the related but non-lethal phenotype $P$. berghei ANKA NK 65 . These results might be of further interest to study the function of hypusine modification with respect to malaria infection and therapy.

\section{Materials and methods}

\section{Ethics statement}

All animal experiments were performed under FELASA category B and GV-SOLAS standards. Animal experiments were approved by German authorities (Regierungspräsidium Karlsruhe, Germany).

siRNAs (small interfering RNAs) for targeting DHS and elF-5A The following double stranded siRNAs were used and synthesized by Ambion, Karlsruhe, Germany: RNAi for 
DHS and EIF5A knockdowns, siRNA duplexes were used which were obtained by annealing the RNA oligonucleotides synthesized by Ambion, Karlsruhe, Germany. The oligonucleotides $(3000 \mathrm{mM}$ for dhs, $1500 \mathrm{mM}$ for eIF-5A) were phosphorylated in a reaction volume of $20 \mu \mathrm{l}$ with 3 Units polynucleotide kinase

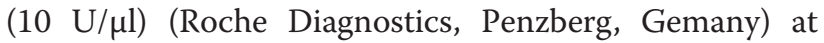
$37^{\circ} \mathrm{C}$ for $45 \mathrm{~min}$. The reaction was stopped on ice for $1 \mathrm{~min}$. An annealing reaction was performed at $95^{\circ} \mathrm{C}$ with subsequent cooling of the reaction to room temperature overnight.

After annealing the siRNA duplexes were cloned into pSilencer 1.0-U6 vector before transfection into $293 \mathrm{~T}$ cells or schizonts.

For the DHS knockdown \#43, RNA oligonucleotides 5'- UGUUAGUGAAGAUCUUAAUtt-3' and 5'-AUUAA GAUCUUCACUAACAtt-3' were applied targeting the nucleotide positions $337-358$ in the plasmodial dhs cDNA. For the DHS knockdown \#176, RNA oligonucleotides 5'- UGAGGAAUGGUGCUGAUUUtt-3' and 5'-AAAUCAGCACCAUUCCUCAtt-3' were applied which targeted nucleotide positions 1269-1290 in the dhs cDNA.

For the eIF-5A knockdowns 4 different siRNA duplexes were generated. For the EIF-5A knockdown \#5, RNA oligonucleotides 5'- ACGGCCACGUGAUG CUAAAtt-3' and 5'- UUUAGCAUCACGUGGCCGUtt-3' were applied targeting nucleotide positions $81-102$ in the P. vivax eIF-5A cDNA. For the EIF-5A knockdown \#6, RNA oligonucleotides 5'- AGGAGCAUCCUUGCAAA GUtt-3' and 5'- ACUUUGCAAGGAUGCUCCUtt-3' were applied which targeted nucleotide positions 99-120; for EIF-5A knockdown \#7, RNA oligonucleotides 5'-AGUG GUAGAUUACUCCACGtt-3' and 5'- CGUGGAGUAAU CUACCACUtt-3' were used for targeting nucleotide positions 115-136. For eIF-5A knockdown \#18, RNA oligonucleotides 5'- CUGAGUUGCAGCUGAUUGAtt-3' and 3'- UCAAUCAGCUGCAACUCAGtt-5' were applied which targeted the $e I F-5 A$ gene at nucleotide positions 163-184.

\section{Construction of pSilencer1.0-U6 vector with double stranded siRNA of DHS and elF-5A}

$20 \mu \mathrm{g}$ of (Ambion/Invitrogen, Karlsruhe, Germany) was double digested with EcoRI/ApaI (20 U) in a reaction volume of $20 \mu \mathrm{l}$ and dephosphorylated with calf intestine alkaline phosphatase (CIP) (MBI Fermentas, St. Leon Rot, Germany) $(1 \mathrm{U} / \mu \mathrm{l})$ for 1 hour at $37^{\circ} \mathrm{C}$. The double digested vector was gel-purified according to the Mini Elute Gel Extraction Kit protocol from Qiagen, (Hilden, Germany). Ligation of the annealed oligos was performed with the ligation kit from Roche Diagnostics, (Penzberg, Germany). Positive constructs were analysed after double digestion with ApaI and HindIII.

\section{Cloning the full length dhs CDNA and elF-5A cDNA into eukaryotic PCDNA3 vector}

Amplification of the $d h s$ gene was performed from the recombinant pet-Blue1 plasmid (Novagen, Darmstadt, Germany) from Plasmodium falciparum with primers containing recognition sites for EcoRI (restriction site is underlined) dhs forward 5'-TTTGAATTCATGGTG GATCACGTTTC-'3' and Not I dhs reverse 5'- TTT GCGGCCGCTCACATATCTTTTTTCCTC- 3'. The resulting fragment of 1491 bp was digested with EcoRI and NotI and ligated into EcoRI/NotI treated pcDNA3 vector (Invitrogen, Darmstadt, Germany) and re-sequenced. For construction of an eIF-5A cDNA containing pcDNA3 vector, the $e I F-5 A$ nucleic acid sequence was amplified from a recombinant plasmid pSTBlue-1 Acceptor ${ }^{\mathrm{Tm}}$ vector (Novagen, Darmstadt, Germany) with primers containing EcoRI eIF-5Aforward 5' -AAA GAA TTC ATG TCA GAC CAC GAA AC-3' and NotI eIF-5Areverse 5'-TTT GCG GCC GCC TAG GAG GAC AAC TCC-3' restriction sites.

\section{Cotransfection of pSilencer1.0-U6 vectors into $293 \mathrm{~T}$ cells}

In a 6 well microtiter plate $7 \times 10^{5} 293 \mathrm{~T}$ cells were seeded in all 6 wells. Four different sets of cotransfections were performed: DHS; i) $P$. falciparum dhs cDNA in pcDNA3 $(0.3 \mu \mathrm{g})$, ii) $P$. falciparum dhs cDNA in pcDNA3 and premade scramble II duplex negative control siRNA $(1.0 \mu \mathrm{g})$, iii) $P$. falciparum dhs cDNA in pcDNA3 and DHS- specific shRNA construct \#43 (1.0 $\mu \mathrm{g})$, iv) P. falciparum dhs cDNA in pcDNA3 and DHS-specific shRNA construct \#176 (1.0 $\mu \mathrm{g})$. The various transfections were mixed with transfection mix (total vol. $400 \mu \mathrm{l}$ ), which contained Opti-MEM ${ }^{\circledR}$ (Invitrogen, Karlsruhe, Germany) and polyethylenimine (PEI) (4 $\mu \mathrm{l} /$ $\mu \mathrm{g}$ ), and were added to the cultures. After $10 \mathrm{~min}$ of incubation at room temperature, the culture supernatants were substituted by $2 \mathrm{ml}$ of DMEM (Dulbecco's Modified Eagle's Medium) (Invitrogen, Karlsruhe, Germany) and the cell cultures were incubated overnight at $37^{\circ} \mathrm{C}$. The next day, medium was changed and supplemented with streptomycin $(60 \mu \mathrm{g} / \mathrm{ml})$. Prior to transfection, the cells were washed with PBS-buffer (phosphate buffer saline).

Cotransfection of $P$. falciparum eIF-5A pcDNA3based expression vector in combination with 4 different sets of siRNA vectors was performed according to a protocol from Invitrogen (Karlsruhe, Germany): i) P. falciparum eIF-5A expression vector $(0.3 \mu \mathrm{g})$ and aquaporin-5 specific-siRNA $(2.7 \mu \mathrm{g})$ ii) $P$. falciparum eIF-5A expression vector $(0.3 \mu \mathrm{g})$ and eIF-5A-specific shRNA construct \#18 $(2.7 \mu \mathrm{g})$, iii) P. falciparum eIF5A expression vector $(0.3 \mu \mathrm{g})$ and eIF-5A-specific shRNA \#6 (2.7 $\mu \mathrm{g})$, iv) P. falciparum eIF-5A expression vector $(0.3 \mu \mathrm{g})$ and eIF-5A shRNA construct \#7 $(2.7 \mu \mathrm{g})$, v) 
P. falciparum eIF-5A expression vector $(0.3 \mu \mathrm{g})$ and eIF5A shRNA \#5 $(2.7 \mu \mathrm{g})$.

\section{Isolation of cellular RNA}

Isolation of total cellular RNA was performed according to the RNeasy Plant Mini Kit (Qiagen, Hilden, Germany). The quality of the isolated RNA was verfied by agarose gel electrophoresis and the quantity and purity was determined by UV spectrometry.

\section{RT-PCR analysis of elF-5A and DHS silencing in vitro and in vivo}

To monitor the silencing of eIF-5A and DHS, RT-PCR was performed according to a protocol from the AccessQuick $^{\mathrm{TM}}$ RT-PCR System (Promega, Mannheim, Germany). For the RT-PCR reaction gene specific primers for eIF-5Aforward 5'ATGTCAGACCACGAAACGT-3'/ eIF-5A reverse 5'-CTAGGAGGACAACTCCTTCACC GC- 3' and dhs forward 5'-ATAGTGCCTAATGA TAATTA -3'/dhs reverse 5'-AACCTCCTCCGAGAA TAATAATACCAG -3' were used. For control, human GAPDH-specific sequences were amplified using the following primers: GAPDH forward 5'-ATGGGGAAG GTGAAGGTCGG-3' and GAPDH reverse 5'-TTACTC CTTGGAGGCCATGTGG-3'. For RT-PCR reactions monitoring cDNA formation in in vivo experiments after P.berghei infection the following $P$. berghei-specific PCR primers were used: $e I F-5 A$ forward 5'-ATGTCA GACCACGAAACGT-3'/eIF5A reverse 5'- TATGATGA CATTTCTTTAAGC-3' and dhs forward 5'-ATGGAT GGGGTATTCAAAGA-3'/dhs reverse 5'-CTAATCACT TTTTTCTCCTTTT-3'. To analyze the quality of the cellular total RNA $i$-tubulin forward 5'-ATGAGA GAAGTAATAAGTAT-3' and $\alpha$-tubulin reverse 5'-TGT TGATAAAACTGAATTAT-3' primers were applied, resulting in a specific $\alpha$-tubulin fragment of $548 \mathrm{bp}$.

Plasmodium transfection using shRNA expressing vectors Parasite transfection using sh expression vectors without Pyrimethamine selection was performed as described in [24].

\section{Preparation of protein extracts from transfected $P$. berghei parasites}

To detect eIF-5A and DHS expression in transfected and wildtype $P$. berghei parasites, intraerythrocytic stages were purified by CF11 Cellulose (Whatman) (Millipore, Schwalbach, Germany) to remove platelets and leukocytes. Parasites were lysed in $0.2 \%$ saponin and resuspended in PBS (LifeTechnologies/Invitrogen, Karlsruhe, (Germany). After determination of the protein concentration by Bradford assay [34], extracts were adjusted to the same protein concentration $(20 \mu \mathrm{g})$ with PBS. Alternatively, for the detection of iNos protein, serum was applied from whole blood without anticoagulant according to a protocol from Proimmune [35].

\section{Western blot analysis}

Western blots were performed using the i-Blot dry blotting device system from Invitrogen (Karlsruhe, Germany) for $5 \mathrm{~min}$ at $5.5 \mathrm{amp}$ and $25 \mathrm{~V}$. Protein extracts from blood stages of transfected parasites were resuspended in 1-fold Nupage buffer (Invitrogen, Karlsruhe, Germany) boiled and loaded onto a 12\% SDS-polyacrylamide gel. Immunodetection was performed according to the protocol from the immunodetection kit from Amersham (Munich, Germany). Polyclonal anti-eIF5A antibodies (Eurogentec, Cologne, Germany) raised against the eIF-5A from $P$. vivax and anti-DHS antibodies against $P$. falciparum DHS were applied in dilutions of 1:1000 and 1:5000, respectively. Previous results had shown that the human DHS protein cross-reacts with the P. berghei DHS protein due to highly conserved regions and an overall amino acid identity of $56 \%$ (see within the results section) [11]. Dilutions of $1: 1000$ and 1:5000 of the antibody raised against the eIF-5A from $P$. vivax were used, since both proteins i.e. eIF-5A from $P$. vivax and P. berghei, share 97\% amino acid identity [11].

\section{Induction of HeLa, jurkat and monomac cells and preparation of protein extracts}

HeLa, Jurkat and Monomac were maintained in Dulbecco's modified Eagle medium (DMEM) (Sigma Aldrich, Munich, Germany), supplemented with $10 \%$ (v/v) fetal bovine serum (FBS), L-glutamine ( $2 \mathrm{mM})$, sodium pyruvate $(1 \mathrm{mM})$ penicillin $(50000 \mathrm{U} / \mathrm{ml})$ and streptomycin $(5 \mathrm{mg} / \mathrm{ml}))$.

For stress induction HeLa, Jurkat or Monomac cells were grown in overnight cultures under starving conditions (i.e. 1\% FCS-containing medium). Thereafter, culture supernatants were substituted by DMEM containing 10\% FCS and cells were further incubated for 3 hours. Finally, cell cultures were exposed for 4 hours to $50 \mathrm{ng} / \mathrm{mL}$ phorbol 12-myristate 13-acetate (PMA) and $1 \mu \mathrm{M}$ of the calcium ionophore ionomycin. Subsequently, the respective cell cultures were washed several times with PBS.

In total $10^{6}-10^{7}$ cells were lysed with CelLytic M solution (Sigma Aldrich, Munich, Germany) for 15 minutes on a rocker platform. The lysed cells were centrifuged at $12,000-20,000 \times \mathrm{g}$ to pellet the cellular debris. The supernatant, containing the cell lysate, were used for further analysis.

\section{Determination of total nitric oxide}

Concentrations of nitric oxide were determined by colorimetric detection according to the kit protocol from Enzo Life Sciences. Nitric oxide is converted to nitrate 
which is reduced to nitrite by the enzyme nitrate reductase followed by the colorimetric detection of nitrite as a coloured azo dye product which absorbs visible light at $560 \mathrm{~nm}$. The determination allows the determination of both nitric oxide products nitrate and nitrite.

\section{Additional file}

Additional file 1: Figure S1. Unsuccessful silencing of parasitic EIF-5A by RNAi in 293T cells and subsequent monitoring by RT-PCR. A cotransfection was performed with: lane 1) EIF-5A-shRNA construct P\# 5; lane 2) EIF-5A-shRNA construct P\#; lane 3) EIF-5A-shRNA construct P\# 7; lane 4) pcDNA3 based plasmodal EIF-5A expression vector; lane 5) $P$. falciparum elF-5A expression vector and aquarin-5 specific siRNA; lane 6) EIF-5A-shRNA construct P\# 18.

\section{Competing interests}

The authors declare that they have no competing interests.

\section{Authors' contributions}

MK was involved in the transfection experiments, AS was responsible for the RT-PCR and Western Blot experiments. AKM and CHS performed the $P$. berghei transfection. BAM and TB were involved in the cloning of siRNA oligonucleotides. AK participated practically in the colorimetric assays and Western Blot experiments, prepared the manuscript and organized financial support, AKM and $\mathrm{JH}$ critically appraised the manuscript. We thank Barbara Langer for excellent technical assistance. All authors read and approved the final manuscript.

\section{Acknowledgements}

The work was supported in main parts by a grant from the German Academic Exchange Service (DAAD) to AK (432/lz (2006).

\section{Author details}

${ }^{1}$ University Duisburg-Essen, Medical Research Centre, Institute of Pharmacogenetics, Hufelandstrasse 55, 45147 Essen, Germany. ${ }^{2}$ Heinrich Pette Institute - Leibniz Institute for Experimental Virology, Martinistrasse 52, 20251 Hamburg, Germany. ${ }^{3}$ Department of Infectious Diseases, Parasitology Unit, University Hospital Heidelberg, Im Neuenheimer Feld 324, 69120 Heidelberg, Germany.

Received: 22 January 2012 Accepted: 31 May 2012

Published: 13 June 2012

\section{References}

1. Hammond SM: Dicing and Splicing. The core machinery of the RNA interference pathway. FEBS Lett 2005, 579:5822-5829.

2. Bernstein E, Caudy AA, Hammond SM, Hannon GJ: Role for a bidentate ribonuclease in the initiation step of RNA interference. Nature 2001, 409:363-366.

3. Nykanem A, Haley B, Zamore PD: ATP requirements and small interfering RNA structure in the RNA interference pathway. Cell 2001, 107:309-321.

4. Baum J, Papenfuss AT, Mair GR, Janse CJ, Vlachou D, Waters AP, Cowman AF, Crabb CJ, Koning-Ward TF: Molecular genetics and comparative genomics reveal RNAi is not functional in malaria parasites. Nucl. Acid Res. 2010, 37(11):3788-3798.

5. Gissot M, Brique S, Refour P, Boschet C, Vaquero C: PfMyb1, a Plasmodium falciparum transcription factor, is required for intra-erythrocytic growth and controls key genes for cell cycle regulation. J Mol Biol 2005, 34:29-42.

6. Kumar R, Adams B, Oldenburg A, Musiyenko A, Barik S: Characterisation and expression of a PP1 serine/threonine protein phosphatase (PfPP1) from the malaria parasite, Plasmodium falciparum: demonstration of its essential role using RNA interference. Malar J 2002, 145:1245-1254.

7. Tuteja R, Pradhan A, Sharma S: Plasmodium falciparum signal peptidase is regulated by phosphorylation and required for intra-erythrocytic growth. Mol Biochem Parasitol 2008, 157:137-147.

8. McRobert L, McConkey GA: RNA interference (RNAi) inhibits growth of $P$. falciparum. Mol. Chem. Parasitol. 2002, 119:273-278.
9. Dasaradhi PV, Mohammed A, Kumar A, Hossain MJ, Bhatnagar RK, Chauhan VS, Malhotra P: A role of falcipain-2, principle cysteine proteases of Plasmodium falciparum in merozoite egression. Biochem Biophys Res Commun 2005, 336:1062-1068.

10. Sijwali PS, Rosenthal PJ: Gene disruption confirms a critical role for the cysteine protease falcipain-2 in haemoglobin hydrolysis by Plasmodium falciparum. Proc. Natl. Acad. Sci. USA 2004, 101:4384-4389.

11. Kaiser A, Gottwald A, Maier W, Seitz HM: Targeting enzymes involved in spermidine metabolism of parasitic protozoa-a possible new strategy for anti-parasitic treatment. Parasitol Res 2003, 91(6):508-516.

12. Njuguna JT, Nassar M, Hoerauf A, Kaiser AE: Cloning, expression and functional activity of deoxyhypusine synthase from Plasmodium vivax. BMC Microbiol 2006, 6:91-96. 16.

13. Maier B, Ogihara T, Trace AP, Tersey SA, Robbins RD, Chakrabarti SK, Nunemaker CS, Stull ND, Taylor CA, Thompson JE, Dondero RS, Lewis EC, Dinarello CA, Nadler JL, Mirmira RG: The unique hypusine modification of elF5A promotes islet beta cell inflammation and dysfunction in mice. J Clin Invest 2011, 20(6):2156-2170.

14. Hauber I, Bevec D, Heukeshoven J, Krätzer F, Horn F, Choidas A, Harrer T, Hauber J: Identification of cellular deoxyhypusine synthase as a novel target for antiretroviral therapy. J Clin Invest 2005, 115(1):76-85.

15. Bevec D, Kappel B, Jaksche H, Csonga R, Hauber J, Klier H, Steinkasserer A: Molecular characterization of a cDNA encoding functional human deoxyhypusine synthase and chromosomal mapping of the corresponding gene locus. FEBS Lett 1996, 378(2):195-198.

16. Hofmann W, Reichart B, Ewald A, Müller E, Schmitt I, Stauber RH, Lottspeich $F$, Jockusch BM, Scheer U, Hauber J, Dabauvalle MC: Cofactor requirements for nuclear export of Rev response element (RRE)- and constitutive transport element (CTE)-containing retroviral RNAs. An unexpected role for actin. J Cell Biol. 2001, 152(5):895-910.

17. Maier B, Tersey SA, Mirmira RG: Hypusine: a new target for therapeutic intervention in diabetic inflammation. Discov. Med. 2010, 10(50):18-23.

18. Zanni GM, Cabrales P, Barkho W, Frangos J, Carvalho L: Exogenous nitric oxide decreases brain vascular inflammation, leakage and venular resistance during Plasmodium berghei ANKA infection in mice. J Neuroinflamm. 2011, 66(Zanni GM, Cabrales P, Barkho W, Frangos J, Carvalho L):1-9. 8

19. Hawkes M, Opoka R, Namasopo S, Miller C, Thorpe KE, Lavery JV, Conroy AL, Liles WC, John CC, Kain KC: Inhaled nitric oxide for the adjunctive therapy of severe malaria. Trials 2011, 12:176.

20. de SA Miranda, Brant F, Machado FS, Rachid MA, Teixera AL: Improving cognitive outcome in cerebral malaria: Insights from Clinical and Experimental Research. Cent Nerv Syst Agents Med Chem 2011, 11(4):285-295. 11.

21. Schofield L, Grau GE: Immunological processes in malaria pathogenesis. Nat Rev Immunol 2005, 5(9):722-735.

22. Specht S, Sarite SR, Hauber I, Hauber J, Görbig UF, Meier C, Bevec D, Hoerauf A, Kaiser A: The guanylhydrazone CNI-1493: an inhibitor with dual activity against malaria-inhibition of host cell pro-inflammatory cytokine release and parasitic deoxyhypusine synthase. Parasitol Res 2008, 102(6):1177-1184.

23. Cohen PS, Nakshatri H, Dennis J, Caragine T, Bianchi M, Cerami A, Tracey KJ: $\mathrm{CNI}-1493$ inhibits monocyte/macrophage tumor necrosis factor by suppression of translation efficiency. Proc Natl Acad Sci U S A 1996, 93(9):3967-3971.

24. Janse CJ, Ramesar J, Waters AP: High-efficiency transfection and drug selection of genetically transformed blood stages of the rodent parasite Plasmodium berghei. Nat Protoc 2006, 1:346-356.

25. Mueller AK, Camargo N, Kaiser K, Andorfer C, Frevert U, Matuschewski K, Kappe SH: Plasmodium liver stage developmental arrest by depletion of a protein at the parasite - host interface. Proc Natl Acad Sci U S A 2005, 102(8):3022-3027.

26. De Miranda AS, Brant F, Machado FS, Rachid MA, Teixeira AL: Improving cognitive outcome in cerebral malaria: insights from clinical and experimental research. Cent Nerv Syst Agents Med Chem. 2011, 1(4):285-295.

27. Mohmmed A, Dasaradhi PV, Bhatnagar RK, Chauhan VS, Malhotra P: In vivo gene silencing in Plasmodium berghei--a mouse malaria model. Biochem Biophys Res Commun 2003, 309(3):506-511. 26

28. Tong Y, Park I, Hong BS, Nedyalkova L, Tempel W, Park HW: Crystal structure of human elF5A1: insight into functional similarity of human elF5A1 and elF5A2. Proteins 2009, 75(4):1040-1050. 
29. Kaiser AE, Gottwald AM, Wiersch CS, Maier WA, Seitz HM: Spermidine metabolism in parasitic protozoa- a comparison to the situation in prokaryotes, plants and fungi. Folia Parasitol. (Praha) 2003, 50(1):3-18

30. Hall N, Karras M, Raine JD, Carlton JM, Kooij TW, Berriman M, Florens L, Janssen CS, Christophides GK, James K, Rutherford K, Harris B, Harris D, Churcher C, Pain A, Quail MA, Ormond D, Doggett J, Trueman HE, Mendoza J, Bidwell S, Rajandream MA, Carucci DJ, Yates JR III, Kafatos FC, Janse CJ, Barrel B, Turner CM, Waters AP, Sinden RE: A comprehensive survey of the Plasmodium life cycle by genomic, transcriptomic, and proteomic analyses. Science 2005, 30(5706):82-86.

31. Templin AT, Maier B, Nishiki Y, Tersey SA, Mirmira RG: Deoxyhypusine synthase haploinsufficiency attenuates acute cytokine signaling. Cell Cycle 2011, 10(7):1043-1049.

32. Yeo TW, Lampah DA, Gitawati R, Tjitra E, Kenangalem E, McNeil YR, Darcy CJ, Granger DL, Weinberg JB, Lopansri BK, Price RN, Duffull SB, Celermajer DS, Anstey NM: Impaired nitric oxide bioavailability and L-argininereversible endothelial dysfunction in adults with falciparum malaria. J. Exp. Med. 2007, 204:2693-2704.

33. Dowling DP, llies M, Olszewski KL, Portugal S, Mota MM, Llinás M, Christianson DW: Crystal structure of arginase from and implications for L-arginine depletion in malarial infection. Biochemistry 2010, 49(26):5600-5608. 6

34. Bradford MM: A rapid and sensitive for the quantitation of microgram quantitites of protein utilizing the principle of protein-dye binding. Analytical Biochem. 1976, 72:248-254.

35. Protocols for the preparation of blood plasma and serum. www.proimmune.com.

doi:10.1186/1471-2180-12-107

Cite this article as: Schwentke et al.: In vitro and in vivo silencing of plasmodial dhs and elf-5a genes in a putative, non-canonical RNAirelated pathway. BMC Microbiology 2012 12:107.

\section{Submit your next manuscript to BioMed Central and take full advantage of:}

- Convenient online submission

- Thorough peer review

- No space constraints or color figure charges

- Immediate publication on acceptance

- Inclusion in PubMed, CAS, Scopus and Google Scholar

- Research which is freely available for redistribution 